\title{
Congestive cardiac failure in a 65-year-old man
}

\author{
HM Mardikar, MH Mardikar, NV Deshpande, AU Mahajan, Rony Mathew
}

LTM Medical College and General Hospital, Sion, Bombay 400022, India

Department of

Cardiology

HM Mardikar

NV Deshpande

AU Mahajan

R Mathew

Department of

Medicine

MH Mardikar

Correspondence to

Dr HM Mardikar, 16/7

Mukand Society,

Gawanpada, Mulund(E),

Bombay 400081, India

Accepted 1 June 1995
A 65-year-old man complained of progressive dyspnoea on exertion for a period of 14 weeks with recent history of orthopnoea. He also gave a history of significant loss of weight $(6.5 \mathrm{~kg}$ in seven months). On physical examination his heart rate was 120 beats/min and blood pressure was $110 / 80 \mathrm{mmHg}$. Jugular venous pressure was raised and pedal oedema was present. Cardiac impulse was just outside the midclavicular line in the 6th intercostal space. Cardiac auscultation revealed first and second heart sounds of normal intensity. Audible S3 gallop was present. Auscultation of the lungs revealed fine bibasilar crepts. Electrocardiogram showed normal sinus rhythm and $T$ wave inversion in leads V1 to V6.

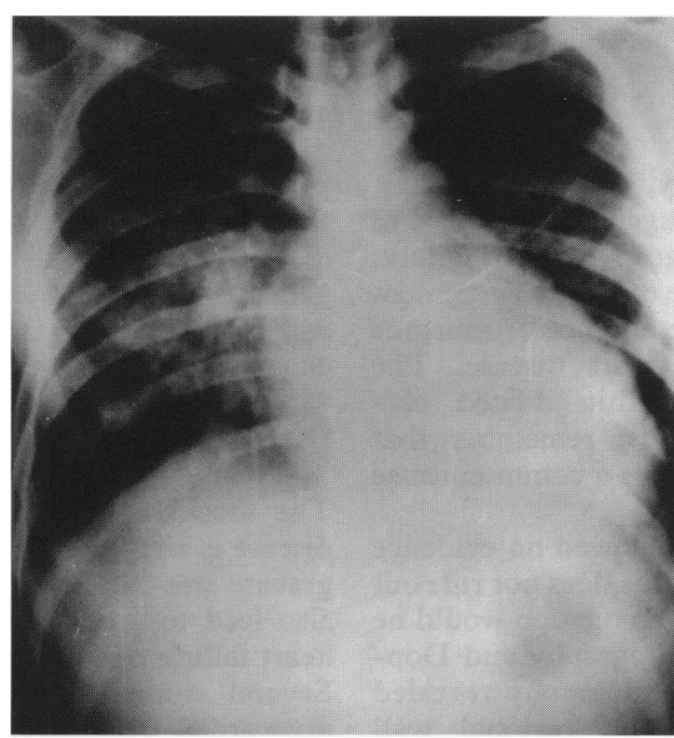

\section{Questions}

1 What is the clinical diagnosis?

2 Name three abnormalities on his chest X-ray

3 How would you treat this patient?

4 How would you investigate this patient?

5 What is the cause of the weight loss?

Figure Chest X-ray, postero-anterior view 
Answers

QUESTION 1

The history is short and simple. The patient is a 65 -year-old man with recent onset congestive heart failure. The abnormalities detected on physical examination all support this diagnosis.

QUESTION 2

Chest radiograph shows moderate cardiomegaly, redistribution of pulmonary venous and arterial flow to upper lobes and upper lobe vessels greater than $3 \mathrm{~mm}$ in diameter in first anterior interspace suggestive of pulmonary venous hypertension. Prominent Kerley's B lines (ie, sharp, linear densities at right base) are suggestive of interstitial pulmonary oedema which indicates raised pulmonary capillary wedge pressure between $20-25 \mathrm{mmHg}$.

\section{QUESTION 3}

The management of such a patient includes removal of the underlying cause, removal of precipitating factors and control of congestive heart failure (box 1).

\section{QUESTION 4}

Correlation of the history, physical examination and investigations suggest a diagnosis of enlarged heart and congestive heart failure. Conditions that can cause cardiac enlargement must be considered. The absence of murmur points against valvular heart disease. The patient may have idiopathic dilated cardiomyopathy but one must remember that ischaemic cardiomyopathy is a common cause of this condition.

The electrocardiogram showed no evidence of $Q$ wave infarction, but this does not rule out ischaemia. So, the next investigation would be two-dimensional echocardiography and Doppler examination. Echocardiography revealed four-chamber dilatation, no regional wall motion abnormality and depressed left ventricular ejection fraction. Thus echocardiography did not make us any wiser. Should such patient be labelled as idiopathic dilated cardiomyopathy or investigated further?

Ischaemic cardiomyopathy is the condition in which coronary artery disease results in severe myocardial dysfunction, with a clinical picture resembling dilated cardiomyopathy. Long-term reduction in myocardial perfusion

\begin{tabular}{|l|}
\hline Control of congestive heart failure \\
\hline Reduction of workload \\
- physical and emotional rest \\
- vasodilators \\
Improvement of cardiac function \\
- digitalis glycosides \\
- other positive inotropic agents \\
Control of excessive salt and water retention \\
- low sodium diet \\
\hline
\end{tabular}

Box 1 may lead to persistent left ventricular dysfunction without tissue necrosis as long as myocardial flow is sufficient to maintain normal contractile function. This is also known as hibernating myocardium. Keeping this in mind, the patient was subjected to cardiac catheterization and coronary angiography which revealed normal coronary arteries, increased left ventricular filling pressures and depressed left ventricular ejection fraction (LVEF) by $25 \%$.

\section{QUESTION 5}

Long-standing heart failure, particularly right ventricular failure, can lead to significant weight loss and cachexia. But in this patient heart failure is of recent onset.

Thyrotoxicosis is one disease which is associated with weight loss. Thyrotoxicosis is known to cause various types of cardiovascular problems like high output cardiac failure, atrial fibrillation and ischaemia. It can also aggravate pre-existing cardiac disease and also, by itself, lead to cardiac disease.

A thyroid function test was suggestive of thyrotoxicosis. Thyroid scan showed diffuse avid uptake of radioiodine suggestive of Graves disease. The patient was immediately put on propylthiouracil and $\beta$-blockers. In the next three months all the symptoms of heart failure disappeared, and there was no audible S3 gallop. The patient was in an euthyroid state and repeated echocardiogram showed normal chamber size and normal LVEF.

\section{Discussion}

The association of thyrotoxicosis and heart disease is well known. Thyrotoxicosis can aggravate pre-existing cardiac disease and can also lead to atrial fibrillation and congestive heart failure or worsening of angina pectoris. ${ }^{1}$ Several studies have documented enhanced myocardial performance in the hyperthyroid state. Indices of myocardial contractility derived from measurement of systolic time intervals have shown a shortened pre-ejection period and isovolumic contraction time which returns to normal after antithyroid therapy. ${ }^{2,3}$ Forfar and co-workers ${ }^{4}$ have shown asymptomatic reversible cardiomyopathy in patients with hyperthyroidism. All these patients had high LVEF at rest but a significant fall during exercise. During the euthyroid state all these patients showed greater LVEF during exercise.

Reversible thyrotoxic cardiomyopathy is rare and only four such cases had been reported in the literature. ${ }^{5}$ Three out of the four patients were in their late $50 \mathrm{~s}$ or $60 \mathrm{~s}$. In all patients congestive heart failure was the first symptom of hyperthyroidism. Two patients had atrial fibrillation. The improvement in ejection fraction with antithyroid treatment was seen as early as $\mathbf{1 8}$ days. In these patients there was full or near complete recovery of left ventricular function.

The clinical importance of the above observation may provide an insight into thyrotoxic dilated cardiomyopathy. It is possible that thyrotoxicosis initially manifests with increased LVEF at rest which may fall 
significantly during exercise. Most patients are treated early for thyrotoxicosis due to other symptoms and this abnormality goes undetected unless specifically sought for. A few patients, particularly in the older age group, may present with predominant cardiac symptoms and a picture of dilated cardiomyopathy with or without atrial fibrillation and history of significant weight loss. The cause of this cardiomyopathy is not clear. Either thyrotoxicosis lowers the threshold for development of cardiomyopathy or it may be a direct effect of increased thyroid hormone. It is important to appreciate that thyrotoxicosis can lead to dilated cardiomyopathy which is partly or fully reversible.

1 Woeber KA. Thyrotoxicosis and heart failure. $N$ Engl $\mathcal{F}$ Med 1992; 327: 94-8.

2 Burckhardt D, Staub JJ, Kraenzlin M, Raeder E, Engel U, Cloppenburg $P$. The systolic time intervals in thyroid dysfunction. Am Heart f 1978; 95: 187-96.

3 Cohen MW, Sculman IC, Spenillo A, Surks MI. Effects of thyroid hormone on left ventricular function in patients treated for thyrotoxicosis. Am $\mathcal{F}$ Cardiol 1981; 48: 33-8.
Idiopathic dilated cardiomyopathy is very common. But before diagnosing such a disease which has no definitive treatment and a dismal prognosis one must make a sincere effort to find any treatable cause that can masquerade as dilated cardiomyopathy.

\section{Final diagnosis}

Thyrotoxic dilated cardiomyopathy (reversible).

Keywords: thyrotoxic cardiomyopathy, congestive heart failure

4 Forfar JC, Muir AL, Sauvers SA, Toft AD. Abnormal left ventricular function in hyperthyroidism. Evidence for a possible reversible cardiomyopathy. N Engl f Med 1982; 307: $1165-70$.

5 Safirstein SM, Santana O, Agasten A. Thyrotoxicosis associated with reversible cardiomyopathy. Am Heart $\mathcal{f}$
1994; 128: $616-7$.

\title{
Cortical blindness in a 35-year-old man
}

\author{
C Rickards, DI Shepherd
}

A 35-year-old man presented with a three-month history of increasing tunnel vision which had progressed to complete blindness. There was also a two-week history of progressive left-sided weakness. Examination revealed cortical blindness, left hemiparesis and crusted impetigo around the mouth. A computed tomography (CT) scan performed 12 weeks after the onset of his visual symptoms is shown below. His full blood count was normal except for an absolute lymphocyte count of $0.7 \times 10^{6} / 1$ (normal range 1.5 to 4 ). Liver function tests were mildly abnormal.

\section{Department of Neurology, North Manchester General Hospital, Crumpsall, Manchester M8 6RB, UK \\ C Rickards \\ DI Shepherd}

Accepted 1 June 1995

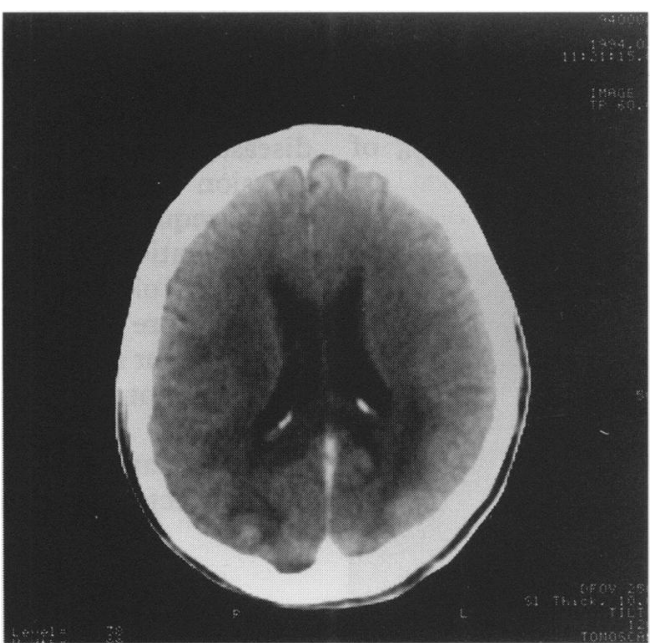

Figure 1 Contrast CT scan showing low attenuation in the white matter of the posterior hemispheres. There is no significant enhancement and no mass effect.

\section{Questions}

1 Name three causes of cortical blindness?

2 What is the most likely underlying diagnosis?

3 What two further investigations are indicated? 